\title{
The Effect Of Dexmedetomidine As Adjuvant To Ropivacaine 0.1\% For Femoral Nerve Block On Strength Of Quadriceps Muscle In Patients Undergoing Total Knee Arthroplasty: A Double-Blinded Randomized Controlled Trial
}

This article was published in the following Dove Press journal: Journal of Pain Research

\author{
Xiaoyu Yang ${ }^{1} *$ \\ Wenbin Kang ${ }^{1} *$ \\ Wei Xiong' \\ Dihan Lu' \\ Zhibin Zhou (D) \\ Xi Chen ${ }^{2}$ \\ Xue Zhou ${ }^{1,3}$ \\ Xia Feng'
}

'Department of Anesthesiology, The First Affiliated Hospital of Sun Yat-Sen University, Guangzhou, People's Republic of China; ${ }^{2}$ Department of

Anesthesiology, Tianjin Medical

University Cancer Institute and Hospital,

Tianjin, People's Republic of China;

${ }^{3}$ MGH Center for Translational Pain Research, Department of Anesthesia, Critical Care and Pain Medicine, Massachusetts General Hospital, Harvard Medical School, Boston, Massachusetts, USA

*These authors contributed equally to this work
Correspondence: Xue Zhou; Xia Feng Department of Anesthesiology, First Affiliated Hospital of Sun Yat-Sen

University, No. 58 Zhongshan 2nd Road, Guangzhou, Guangdong 51 0080, People's Republic of China

Tel +86-20-87332200-8273

Fax +86-020-873355766

Email zhoux77@mail.sysu.edu.cn;

fengxia@mail.sysu.edu.cn
Background: Femoral nerve block (FNB) has been considered as an excellent analgesic modality in total knee arthroplasty (TKA) pain control. However, relatively high concentration of ropivacaine could lead to quadriceps muscle weakness and increase the risk of postoperative falls.

Objective: This double-blinded randomized controlled study was designed to investigate the effect of a combination of dexmedetomidine with a lower concentration of ropivacaine on quadriceps muscle strength and analgesic effect in FNB.

Methods: A total of 90 patients scheduled for TKA were randomized to receive continuous FNB postoperatively using $0.2 \%$ ropivacaine (H group), $0.1 \%$ ropivacaine ( $\mathrm{L}$ group) or $0.1 \%$ ropivacaine combined with $2 \mu \mathrm{g} / \mathrm{kg}$ dexmedetomidine (LD group). Meanwhile, intravenous patient-controlled analgesia with morphine was administered to patients. The primary endpoint was the strength of quadriceps muscle evaluated by manual muscle testing (MMT) and Timed Up and Go test (TUG). The secondary endpoint was the pain scores and morphine consumption among different groups.

Results: For MMT, LD group showed higher quadriceps muscle strength than the other two groups $(P<0.05)$ at $12 \mathrm{hrs}$ postoperatively. TUG test was conducted to measure the walking ability, and showed that scores were significantly better in LD group than those in $\mathrm{H}$ group and L group $(P<0.05)$ at 24 and $48 \mathrm{hrs}$ postoperatively. There was no significant difference between $\mathrm{H}$ and LD group in the numeric rating scales (NRS) scores both at rest and at $45^{\circ}$ flexion. The total morphine consumption in L group was significantly higher than in H or LD group $(P<0.001)$.

Conclusion: Collectively, the addition of dexmedetomidine $2 \mu \mathrm{g} / \mathrm{kg}$ to $0.1 \%$ ropivacaine preoperatively would preserve quadriceps muscle strength with satisfactory analgesia in patients undergoing TKA. (This study was registered at ClinicalTrials.gov, identifier NCT03658421).

Keywords: femoral nerve block, dexmedetomidine, total knee arthroplasty, postoperative pain, muscle strength

\section{Introduction}

Due to the prevalent mild or severe post-operative pain in most patients undergoing total knee arthroplasty (TKA), multimodal analgesia plays an important role to effectively relieve pain, which involves a combination of different techniques and 
analgesic agents. ${ }^{1}$ In recent years, the pain management protocols during the early postoperative period have shifted toward effective analgesia with limited motor involvement due to the emphasis on faster recovery following TKA.

Among all the modalities, continuous femoral nerve block (FNB) providing excellent postoperative analgesia, is often considered as the gold standard for TKA pain relief. ${ }^{2,3}$ However, patients receiving continuous FNB generally suffer from substantially decreased quadriceps muscle strength, which increases the risk of postoperative falls. $^{4-6}$ Injection of a relatively lower concentration of local anesthetics around the femoral nerve will theoretically exert less influence on quadriceps strength. Previous studies comparing equal volumes of different concentrations of local anesthetics in continuous femoral nerve block have shown that adequate analgesia is likely to be achieved with concentrations of ropivacaine between $0.1 \%$ and $0.2 \%$, which, however, still lack robust mobility data. ${ }^{7}$

Over recent years, the addition of multiple types of additives to local anesthetics has been reported to improve analgesic effect of peripheral nerve block. ${ }^{8,9}$ Dexmedetomidine, an $\alpha_{2}$-agonist with an eight-fold affinity for $\alpha_{2}$-adrenergic receptors (sedate and analgesic effects) as clonidine, while exerts much less $\alpha_{1}$-effects. ${ }^{10}$ Multiple randomized controlled trials have been conducted to examine its effectiveness as a peripheral nerve block adjuvant, revealing that dexmedetomidine could prolong the duration of analgesia. ${ }^{11,12}$ However, there are still no studies evaluating the effect of femoral nerve block with or without dexmedetomidine as adjuvant on the strength of the quadriceps muscle after TKA. Moreover, to the best of our knowledge, there have been no studies that directly investigated the effect of a combination of dexmedetomidine with a lower concentration of ropivacaine on the balance of analgesia with mobility after TKA.

To this end, in our study, we hypothesized that the addition of dexmedetomidine at a concentration of $2 \mu \mathrm{g} / \mathrm{kg}$ to $0.1 \%$ ropivacaine in FNB would intensify postoperative analgesia and further preserve quadriceps muscle strength in patients undergoing TKA. The primary aim of this study was to investigate the strength of the quadriceps muscle by manual muscle testing (MMT) and Timed Up and Go test (TUG). ${ }^{6}$ The secondary endpoint was to record the pain score and morphine consumption among different groups. Hence, we aimed to determine a better protocol for FNB as a main part of analgesic approach in patients receiving TKA.

\section{Materials And Methods}

\section{Patient Selection}

This randomized, single-center, double-blind clinical trial was conducted after the approval from the Ethics Committee of the First-affiliated Hospital of Sun Yat-sen University (No. [2018]45). The trial complied with the Declaration of Helsinki and was registered at ClinicalTrials.gov (identifier NCT03658421). Written informed consent was obtained from all patients willing to participate in this study. From September 1, 2018 to November 5, 2018, a total of 90 patients scheduled for TKA with American Society of Anesthesiology (ASA) I-II, aged 50-80 years old were included in this trial. Exclusion criteria included refusal to participate, unicompartmental knee arthroplasty, body mass index greater than $35 \mathrm{~kg} / \mathrm{m}^{2}$, cognitive or psychiatric history that may interfere in assessment, refusal to general anesthesia, contraindications to laryngeal mask airway insertion under general anesthesia or peripheral nerve blocks (localized infections, sepsis, coagulopathy, bleeding diathesis or preexisting lower extremity neurological abnormality) and allergy to the drugs used.

\section{Randomization}

A computer-generated sequence of random numbers was used to randomize the study participants on a $1: 1: 1$ ratio with no restrictions to receive three different local anesthetic regimens for FNB. The randomization sequence was generated at the coordinating center, before the start of the trial, using automated web-based software (Random Allocation Software). The allocation was concealed in sealed opaque sequentially numbered envelopes kept by another researcher. For each patient, one envelope was handed to the attending regional anesthesiologist or supervised regional anesthesia fellow who was assigned to the block procedure room on the day of surgery and set to perform all nerve blocks required for the current study.

\section{Study Design}

The investigation was designed to study clinically relevant muscle strength and analgesic effect after three different local anesthetic regimens for FNB before and after TKA. To explore the role of local anesthetic concentration, the first regimen comprised of a single bolus of $20 \mathrm{~mL}$ of $0.2 \%$ ropivacaine (high concentration group or $\mathrm{H}$ group) before surgery, followed by continuous femoral nerve block using an infusion of $0.2 \%$ 
ropivacaine $(\mathrm{n}=30)$, whereas the second regimen included $20 \mathrm{~mL}$ of $0.1 \%$ ropivacaine (low concentration group, or L group) before surgery, followed by a continuous femoral nerve block using an infusion of $0.1 \%$ ropivacaine $(n=30)$. To evaluate the role of dexmedetomidine as the adjuvant, the third regimen consisted of $20 \mathrm{~mL}$ solution of $0.1 \%$ ropivacaine added to $2 \mu \mathrm{g} / \mathrm{kg}$ dexmedetomidine (low concentration group with dexmedetomidine, or LD group) with a continuous infusion of $0.1 \%$ ropivacaine $(n=30)$. All the continuous femoral nerve block infusion regimens in the pumps deliberately contained different concentrations of ropivacaine $(0.1 \%$ in L group and LD group, while $0.2 \%$ in $\mathrm{H}$ group) and were started immediately after surgery with an infusion rate of $5 \mathrm{~mL}$ per hour. All local anesthetic solutions administered were prepared by the hospital pharmacy in identical size syringes and infuscate bags. Surgeons and anesthesiologists were blinded to different regimens.

\section{Anesthesia Procedure}

Patients were fasted for $8 \mathrm{hrs}$ before surgery. Routine monitoring with electrocardiography, oxygen saturation, and non-invasive blood pressure was started on arrival to operation room, followed by the successful establishment of peripheral venous access. All FNBs were performed before general anesthesia by the same anesthesiologist in the post-anesthesia care unit (PACU) who had much experience and expertise in ultrasound-guided peripheral nerve block. The in-plane lateral to medial approach was used for ultrasound-guided FNB. First, femoral artery was palpated in the inguinal area. The tip of the needle was located $1-1.5 \mathrm{~cm}$ lateral to the femoral artery pulsation under ultrasound guidance (X-PORTE; SonoSite Inc., USA). Afterwards, a 20-gauge catheter (Perifix; B. Braun Melsungen, Germany) was inserted for continuous nerve block. Patients were subsequently randomized to receive one of the three single local anesthetic infusion regimens in PACU. To be specific, patients in $\mathrm{H}$ group received $20 \mathrm{~mL}$ of $0.2 \%$ ropivacaine through the continuous femoral nerve block (CFNB) catheter. Patients in $\mathrm{L}$ group were administered with $20 \mathrm{~mL}$ of $0.1 \%$ ropivacaine, while patients in LD group received $0.1 \%$ ropivacaine added to $2 \mu \mathrm{g} / \mathrm{kg}$ dexmedetomidine.

After the administration of the initial loading dose, all patients were sent into the operating room and received general anaesthesia. Propofol $(2.5 \mathrm{mg} / \mathrm{kg})$, sufentanil $(0.5$ $\mu \mathrm{g} / \mathrm{kg})$, and cis-atracurium $(0.2 \mathrm{mg} / \mathrm{kg})$ were administered for induction. After induction, a laryngeal mask airway
(LMA, LMAUnique, Singapore) was used for maintenance of the airway. After surgery, all patients received multimodal analgesia of $200 \mathrm{mg}$ celecoxib every $12 \mathrm{hrs}$. The pumps for peripheral nerve block were connected to CFNB catheter with different regimens. Intravenous patient-controlled analgesia (IV-PCA) pumps, including $50 \mathrm{mg}$ morphine diluted in $50 \mathrm{~mL}$ saline (bolus, $2 \mathrm{mg}$; lock-out time, 15 mins), was started in the event of inadequate pain control. All the pumps used for postoperative analgesia were left on patients for $48 \mathrm{hrs}$. The total usage of morphine could be recorded by the pump and sent to a central computer.

\section{Measurements}

The infusion of continuous nerve block was ceased approximately $2 \mathrm{hrs}$ before the measurements, and was further continued after the assessments. The measurements were performed by the same doctor who was blinded to the group assignment. The following measures were recorded at 24 and $48 \mathrm{hrs}$ after surgery around 09:00-18:00.

The MMT was performed in the sitting position with the patients extending their knee against gravity from a flexed position. The grading was recorded from 0 to 5 . To be specific, if the patient was unable to generate any contraction, the test was scored as 0 out of 5 . If the patient was capable of extending the knee against resistance to full extension, the score would be recorded as 3 out of 5 . If the patient was able to hold the knee in extension against gravity, the score would be 5 out of 5 . Moreover, the grading 0 to 2 out of 5 was evaluated according to the degree of the ability of these patients to move a limb (gravity eliminated) throughout the range of motion. MMT was measured at 12, 24, 48, and $72 \mathrm{hrs}$ after surgery.

Walking ability was assessed using a validated ambulation test, the Timed Up and Go (TUG) test. TUG test is able to assess the patient's mobility that requires both balance and static, under the facilitation of any assisting device. In TUG test, patients are asked to rise from a chair, walk to a line 3 meters away, turn around, walk back to the chair, and sit down. The time duration is measured in seconds, which was determined at 24, 48, and $72 \mathrm{hrs}$ after surgery. For both MMT and TUG, the primary endpoint regarding timing was $48 \mathrm{hrs}$ after surgery, when we stopped the pumps and patients started their functional exercise. Notably, great attention should be paid to avoid falls in patients. 
Moreover, the numeric rating scales (NRS) scores at rest and at $45^{\circ}$ flexion of the knee were recorded to evaluate the postoperative pain control. The numeric scale ranges from 0 to 10 , representing from "no pain" to "worst pain imaginable", respectively. Patients were asked to select a number (0-10 integers) to reflect the pain level, which was assessed at $6,12,24,36,48$, and $72 \mathrm{hrs}$ after surgery. In addition, the analgesic consumption and complications of peripheral nerve block were also evaluated in all patients.

\section{Sample Size}

Based on NRS data of a pilot trial conducted in the beginning (10 subjects per group), anticipated sample size was calculated by the PASS software (version 11; NCSS, Tennessee). Tests for two means for a repeated design was adopted and a maximal sample size of 24 subjects per group would be appropriate to have a $90 \%$ power to detect a mean difference in NRS scores. A total of 90 subjects would be enrolled in case of $20 \%$ dropouts.

\section{Statistical Analysis}

Data were analyzed using Statistical Package for the Social Sciences (SPSS) version 23.0 (IBM Corp., NY, USA). Normality would be confirmed first when analyzing numerical data like the MMT scores, NRS scores, and analgesic consumption. Analysis of variance (ANOVA) or nonparametric test (Kruskal-Wallis test) was adopted to compare the effects of the treatments, according to the distribution of the variable. ANOVA for repeated measurements was used to analyze NRS, MMT, and TUG results, followed by Mauchly's test. Categorical data were analyzed using $\chi^{2}$ or Fisher's exact test as appropriate. Bonferroni method was used in correction for multiple pairwise comparisons where is needed. The $\alpha$ level was 0.05 and $P<0.05$ was considered significant.

\section{Results}

A total of 90 patients were assessed for eligibility, 2 patients did not meet the inclusion criteria, and 6 patients refused general anesthesia. Data were analyzed on 30 patients in each group, as shown in Figure 1. The characteristics of patients, preoperative NRS, preoperative mechanical femorotibial angle, preoperative muscle power, and operation duration in $\mathrm{H}$ group, L group, and LD group are shown in Table 1. The baseline characteristics and perioperative variables were similar among the three groups and no complications of peripheral nerve block were reported. ANOVA for repeated measures was used to analyze the data of MMT scores and TUG values. Treatment effects of 3 groups were different from each other $(P<0.001)$. At 12 $\mathrm{h}$ postoperatively, the quadriceps muscle strength was higher in $\mathrm{LD}$ group than $\mathrm{H}$ group and $\mathrm{L}$ group $(P<0.05)$. No differences were found among the three groups at $24 \mathrm{~h}, 48$ $\mathrm{h}$, and $72 \mathrm{~h}$ postoperatively (Figure $2 \mathrm{~A}$ ).

As for TUG values, the sphericity assumption was not violated by Mauchly's test $(P=0.453)$. Walking ability measured by the TUG test was significantly different from each other $(P<0.001)$ and the value of $H$ group was larger than those of L group and LD group at 24 and $48 \mathrm{~h}$ postoperatively $(P<0.05$, Figure $2 \mathrm{~B})$. No significant difference was found among the three groups at $72 \mathrm{~h}$ postoperatively (Figure $2 \mathrm{~B}$ ).

ANOVA for repeated measures was used to analyze the NRS scores at rest. Sphericity was identified by Mauchly's test $(P=0.509)$ and the mean values of the NRS scores at rest in L group were higher than $\mathrm{H}$ or LD group $(P<0.001)$. Patients in H group and LD group reported reduced NRS scores at rest compared with L group at 12,36 , and $48 \mathrm{~h}$ postoperatively $(P<0.5)$. No significant difference was observed among the three groups at $72 \mathrm{~h}$ postoperatively (Figure $3 \mathrm{~A}$ ).

NRS scores at $45^{\circ}$ flexion were also analyzed by ANOVA for repeated measures. Sphericity assumption was identified $(P=0.428)$ and similarly, the mean values of the NRS scores at flexion in L group were higher than $\mathrm{H}$ or LD group $(P<0.001)$. The results indicated the NRS scores at flexion in $\mathrm{H}$ group and $\mathrm{LD}$ group were both lower than in L group at 48 and $72 \mathrm{~h}$ postoperatively $(P<0.05)$. There was no significant difference among the three groups at $24 \mathrm{~h}$ and $36 \mathrm{~h}$ postoperatively (Figure $3 \mathrm{~B}$ ).

Furthermore, the total morphine consumption within $48 \mathrm{hrs}$ after FNB was statistically different as indicated by ANOVA $(P=0.007)$. The mean morphine consumption in L group was larger than those in L group and LD group $(P<0.05)$. No significant difference was found between $\mathrm{H}$ group and LD group (Figure 4).

\section{Discussion}

The objectives of our study were to determine whether the addition of dexmedetomidine to a lower concentration of ropivacaine in preoperative FNB would intensify analgesia and preserve quadriceps muscle strength. As a result, our present outcomes indicated that addition of dexmedetomidine $2 \mu \mathrm{g} / \mathrm{kg}$ to $0.1 \%$ ropivacaine in FNB would preserve quadriceps muscle strength compared to $0.2 \%$ ropivacaine. Moreover, a combination of dexmedetomidine with $0.1 \%$ 


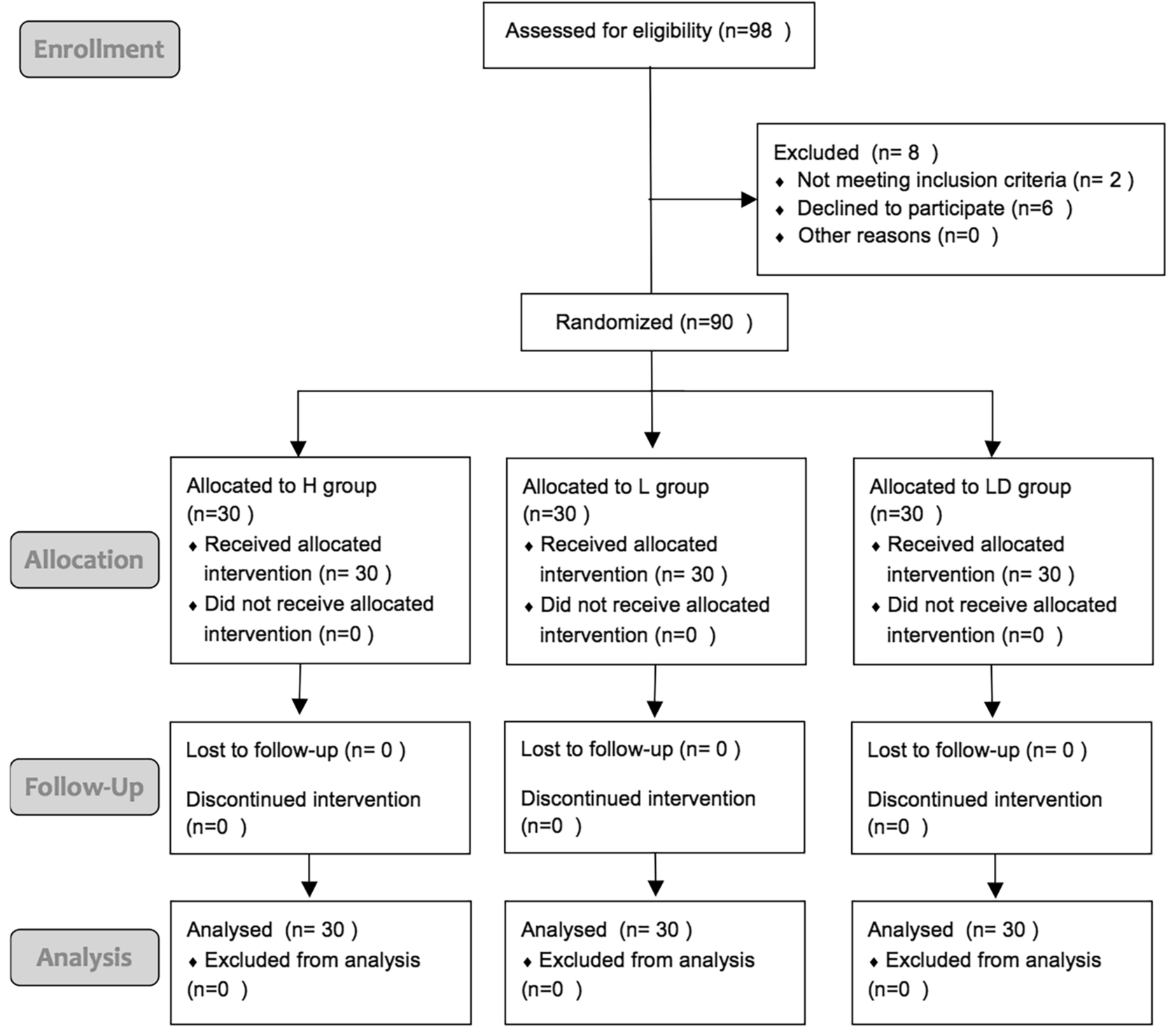

Figure I Consolidated Standards of Reporting Trials (CONSORT) flow diagram showing patient progress through the study phases.

Abbreviations: $\mathrm{H}$ group, high concentration group; L group, low concentration group; LD group, low concentration with dexmedetomidine group.

ropivacaine decreased postoperative pain both at rest and movement, and attenuated opioid consumption analgesia, in comparison with those administered $0.1 \%$ ropivacaine alone.

Post-TKA pain leads to knee stiffness and inhibits early mobilization, which is considered as the most limiting factor for early ambulation and rehabilitation. FNB, a main part of analgesic approach in patients receiving TKA, has been proven to be an effective method to relieve post-TKA pain. ${ }^{2}$ However, the administration of FNB would generally cause substantially decreased quadriceps muscle strength in patients. Moreover, quadriceps weakness could limit patients' ability to participate in any physical rehabilitation program and even place them at high risk of postoperative falling. ${ }^{5}$ Previous attempts have been made to decrease the dose of local anesthetics used in FNB in order to promote the fast recovery speed of patients following TKA. However, a previous study demonstrated no benefit in patients' postoperative rehabilitation and analgesia when using ropivacaine less than $0.1 \%{ }^{13}$ Another research revealed similar analgesic quality between $0.2 \%$ ropivacaine and $0.3 \%$ ropivacaine for postoperative continuous $\mathrm{FNB}^{7}$ Therefore, according to previous studies, it is speculated that, for continuous nerve blocks, local anesthetic concentration and volume might not influence nerve block characteristics, while local anesthetic dose (mass) is the primary determinant 
Table I Patient Demographics And Baseline Characteristics

\begin{tabular}{|c|c|c|c|c|}
\hline Variable & H Group & L Group & $\begin{array}{l}\text { LD } \\
\text { Group }\end{array}$ & $P$-value \\
\hline $\begin{array}{l}\text { Sex (Male/ } \\
\text { female) }\end{array}$ & $42 / 48$ & $39 / 51$ & $46 / 44$ & n.s. \\
\hline Age (Years) & $68.9(2.8)$ & $67.8(3.6)$ & $68.4(2.2)$ & n.s. \\
\hline Weight (kg) & $67.9(5.5)$ & $67.7(4.7)$ & $69.7(4.6)$ & n.s. \\
\hline Height $(\mathrm{cm})$ & $162.6(9.1)$ & $161.8(6.8)$ & I64.I (6.5) & n.s. \\
\hline $\begin{array}{l}\text { Preoperative } \\
\text { NRS }\end{array}$ & $2.3(0.9)$ & $2.4(1.0)$ & $2.6(0.9)$ & n.s. \\
\hline $\begin{array}{l}\text { Preoperative } \\
\text { mechanical } \\
\text { femorotibial } \\
\text { angle }\left({ }^{\circ}\right)\end{array}$ & I74.6 (3.9) & $176.6(1.9)$ & $177.0(1.9)$ & n.s. \\
\hline $\begin{array}{l}\text { Preoperative } \\
\text { muscle power }\end{array}$ & $5(4-5)$ & $5(4.5-5)$ & $5(4-5)$ & n.s. \\
\hline $\begin{array}{l}\text { Operation } \\
\text { duration (min) }\end{array}$ & II 7.7 (8.4) & II9.I (8.3) & $120.4(7.8)$ & n.s. \\
\hline
\end{tabular}

Notes: Continuous data are presented as mean (SD) or median $\left(P_{25}-P_{75}\right)$. There were no statistical differences among all three groups. $(P<0.05)$.

Abbreviations: $\mathrm{H}$ group, high concentration group; $\mathrm{L}$ group, low concentration group; LD group, low concentration with dexmedetomidine group; n.s., not significant.

of perineural infusion effects. ${ }^{14}$ But until now, the most effective ropivacaine concentration for femoral infusion before and after TKA has not been defined.

In our experience, the continuous FNB using an infusion of $0.2 \%$ ropivacaine yielded better analgesic effect than using $0.1 \%$ ropivacaine, which was also shown in our results. Sharma et al found that the addition of perineural dexmedetomidine to $0.2 \%$ ropivacaine significantly improved the quality and duration of post-operative
A

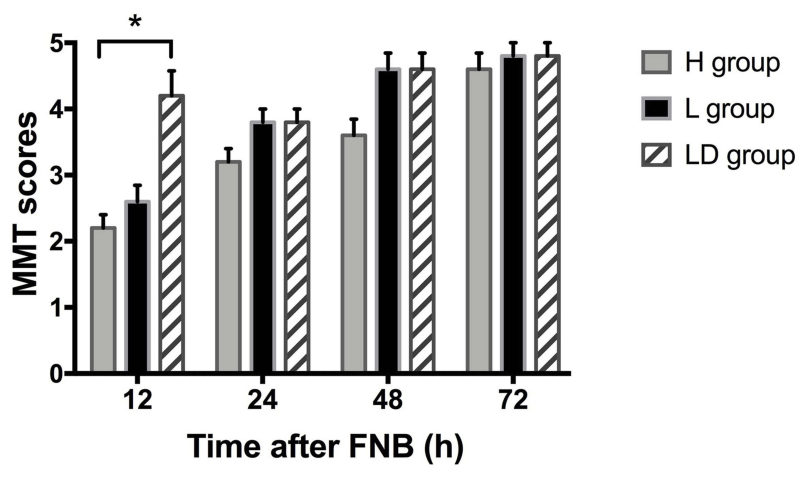

B

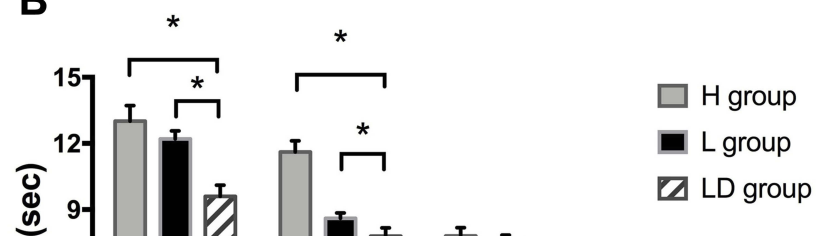

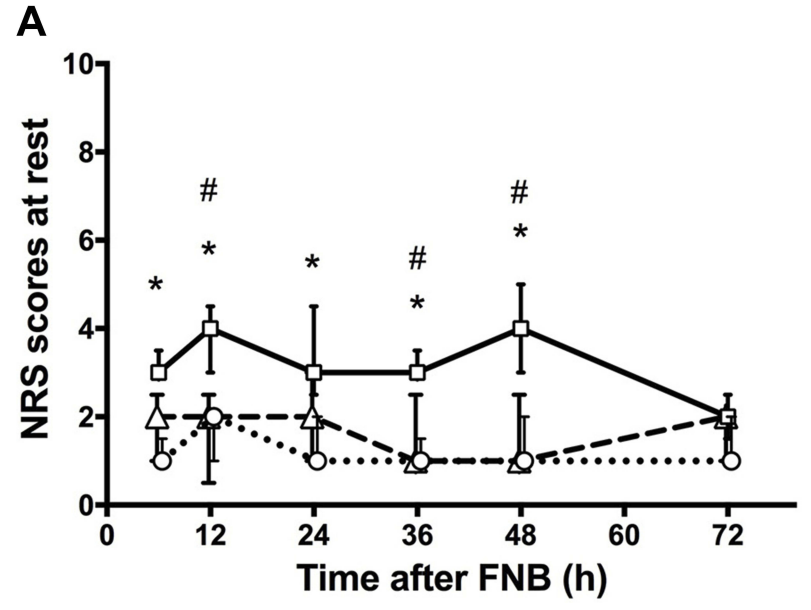

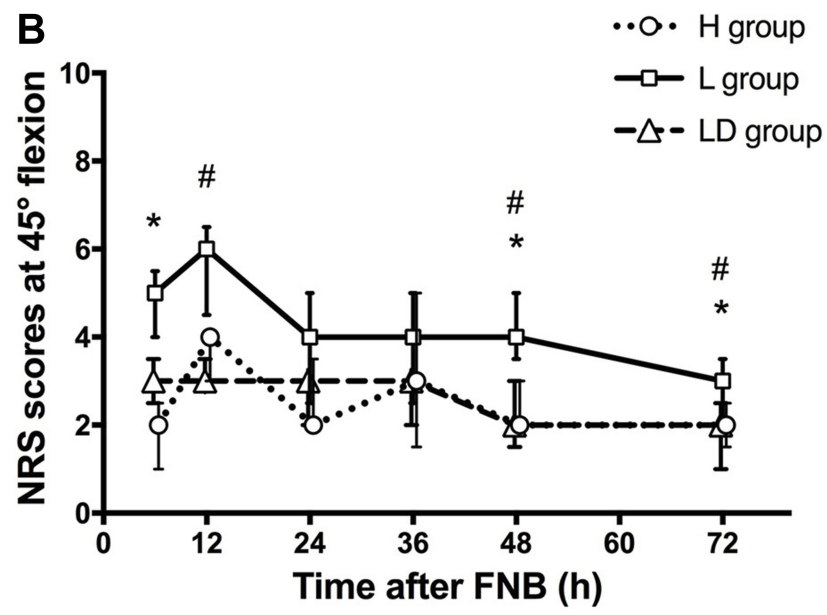

Figure 3 Mean NRS pain scores at rest $(\mathbf{A})$ and at $45^{\circ}$ flexion (B). Data are shown by median and interquartile range (IQR). A P-value less than 0.05 was considered to be statistically significant. $* P<0.05$ compared with $\mathrm{H}$ group; \#, $\mathrm{P}<0.05$ compared with LD group.

Abbreviations: $\mathrm{H}$ group, high concentration group; L group, low concentration group; LD group, low concentration with dexmedetomidine group. 


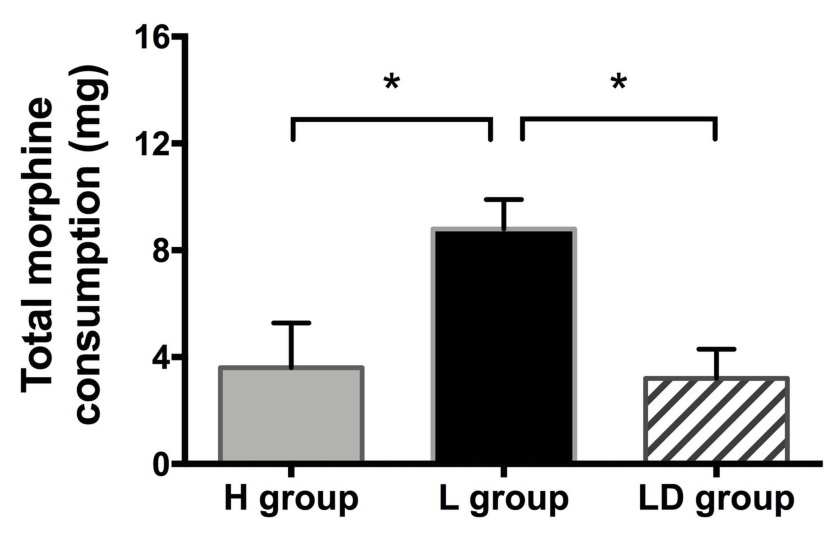

Figure 4 Morphine consumption during 48 hrs after FNB. Total morphine consumptions are presented by median and IQR. $* P<0.05$.

Abbreviations: $\mathrm{H}$ group, high concentration group; L group, low concentration group; LD group, low concentration with dexmedetomidine group.

Perineural dexmedetomidine has been proven to enhance sensory and analgesic block characteristics and exhibit a facilitatory effect when administered intrathecally as part of nerve block. ${ }^{16,17}$ Therefore, in the present study, dexmedetomidine was added to $0.1 \%$ ropivacaine in FNB. In spite of the existing concern regarding the application of dexmedetomidine in FNB, no information about quadriceps muscle strength has ever been reported. Our study not only focused on post-operative analgesia, but also concerned quadriceps muscle strength. During the operations, the surgeons did not perform any local infiltration analgesia technique, and we did not apply any NSAIDs drugs, but we used both continuous peripheral nerve block and the morphine pump as multimodal analgesia strategy, which had been proven sufficient for knee analgesia. Moreover, we tried to avoid interference of other factors except FNB in our study.

Of note, the most important finding of our study is that quadriceps muscle strength was significantly greater in dexmedetomidine $2 \mu \mathrm{g} / \mathrm{kg}$ combined with $0.1 \%$ ropivacaine group than that in $0.2 \%$ ropivacaine group, without major differences in the NRS scores under movement or analgesic consumption (Figures 3 and 4). Moreover, we found that $0.1 \%$ ropivacaine exerted less analgesic effect than $0.2 \%$ ropivacaine or $0.1 \%$ ropivacaine combined with dexmedetomidine. We have assumed that a lack of analgesic effect of $0.1 \%$ ropivacaine could lead to weak TUG results. Intriguingly, we believe that with the application of intravenous morphine, patients would have better 48 h-recovery after surgery and show improved TUG results. In our clinical setting, the addition of dexmedetomidine to a lower concentration of ropivacaine in FNB could decrease the motor block of quadriceps muscle in patients undergoing TKA. During the study, we stopped the pump at $48 \mathrm{hrs}$ after surgery according to our clinical protocol for two reasons. Firstly, NRS scores measured at this time point in patients were less than 4 , indicating effective analgesia. Secondly, the patients had to start leaving their beds for functional exercise, so the pumps had to be stopped.

The mechanism of dexmedetomidine acting perineurally has not been well studied. Like clonidine, dexmedetomidine can enhance the degree of hyperpolarization by blocking the Ih current (generated by low-grade stimulation and activation of $\mathrm{Na}^{+} / \mathrm{K}^{+}$pump). ${ }^{10}$ Animal studies have shown that the addition of dexmedetomidine to local anesthetic would increase the duration of sensory and motor blockade in a rat model of sciatic nerve blockade. ${ }^{18} \mathrm{~A}$ study has been performed to compare two different doses of dexmedetomidine, 1 and $2 \mu \mathrm{g} / \mathrm{kg}$ respectively, along with $0.25 \%$ bupivacaine used alone in FNB for postoperative analgesia after TKA. Consequently, they found that dexmedetomidine at $2 \mu \mathrm{g} / \mathrm{kg}$ dose in FNB showed better analgesic effect than that at $1 \mu \mathrm{g} / \mathrm{kg}$ after TKA. However, they only evaluated the effect of dexmedetomidine in providing analgesia, but failed to assess early ambulation. ${ }^{19}$ Another study showed that the addition of dexmedetomidine $1 \mu \mathrm{g} / \mathrm{kg}$ to $0.25 \%$ bupivacaine in thoracic paravertebral blocks (PVB) in patients undergoing modified radical mastectomy improved the quality and the duration of analgesia, and also provided an analgesic sparing effect without any serious side effects. ${ }^{20}$ Thus, in our study, the dosage of $2 \mu \mathrm{g} / \mathrm{kg}$ dexmedetomidine was selected as adjuvant to ropivacaine. Similar to previous studies, dexmedetomidine showed no adverse cardiovascular responses.

Here, we performed MMT to evaluate the function and strength of individual muscles/muscle groups based on manual resistance and the forces of gravity. MMT results depend on the subjective judgement of investigators, which is difficult to precisely measure because of postoperative pain. ${ }^{21}$ In our study, we found L group showed worse MMT results than LD group at $12 \mathrm{~h}$ after surgery, indicating that $0.1 \%$ ropivacaine has less analgesic effect than $0.2 \%$ ropivacaine or $0.1 \%$ ropivacaine combined with dexmedetomidine. However, at 24 $\mathrm{h}$ after surgery, this phenomenon disappeared. We assumed that patients in L group used morphine to relieve the pain. Moreover, TUG test was performed to assess the walking ability, and postoperative pain might also affect walking ability, which explained the causes for the worse walking ability in L group. TUG is known as a simple, quick, and clinical performance-based measure of lower extremity function, 
mobility, and fall risk. ${ }^{22}$ TUG tests have been used in many TKA trials. One study aimed to investigate the continuous adductor canal block versus CFNB after TKA for mobilization capability. They administered continuous infusion of $0.2 \%$ ropivacaine to patients without any intravenous morphine pumps. Because the concentration of ropivacaine they applied was higher than that in our study, the TUG scores in their study were much higher than ours. ${ }^{23}$ Jaeger et al reported a similar TUG score level while they were trying to find out the relationship between FNB and quadriceps strength. ${ }^{24}$ They used a similar ropivacaine concentration as that in our study. All of these indicated that TUG scores are highly associated with concentration of local anesthetics used.

In our study, we assumed that $2 \mu \mathrm{g} / \mathrm{kg}$ dexmedetomidine used in FNB presents better analgesic effect. Thus, a lower concentration of ropivacaine combined with $2 \mu \mathrm{g}$ / $\mathrm{kg}$ dexmedetomidine as adjuvant could decrease the motor block of quadriceps muscle in patients undergoing TKA, which is a better protocol for FNB in patients.

\section{Ethics Approval And Consent To Participate}

This trial was approved by the Ethics Committee of the First-affiliated Hospital of Sun Yat-sen University (No. [2018]45). Written informed consent was obtained from all patients willing to participate in this study.

\section{Availability Of Data And Materials}

The data were part of dataset of Department of Anesthesiology, First-affiliated Hospital, Sun Yat-sen University, which is not public presently. The authors do not intend to share individual deidentified participant data.

\section{Acknowledgements}

We appreciate Professor Lihua Zhou, from the Department of Anatomy, Zhong Shan Medical College, for her detailed instruction of the clinical anatomy.

\section{Author Contributions}

All authors contributed to data analysis, drafting or revising the article, gave final approval of the version to be published, and agree to be accountable for all aspects of the work.

\section{Funding}

This work was supported by research grants from the National Natural Science Foundation of China (81571032; 81701047; 81870829).

\section{Disclosure}

The authors report no conflicts of interest in this work.

\section{References}

1. Bauer MC, Pogatzki-Zahn EM, Zahn PK. Regional analgesia techniques for total knee replacement. Curr Opin Anaesthesiol. 2014;27 (5):501-506. doi:10.1097/ACO.0000000000000115

2. Paul JE, Arya A, Hurlburt L, et al. Femoral nerve block improves analgesia outcomes after total knee arthroplasty: a meta-analysis of randomized controlled trials. Anesthesiology. 2010;113(5):11 44-1162. doi:10.1097/ALN.0b013e3181f4b18

3. Choy WS, Lee SK, Kim KJ, Kam BS, Yang DS, Bae KW. Two continuous femoral nerve block strategies after TKA. Knee Surg Sports Traumatol Arthrosc. 2011;19(11):1901-1908. doi:10.1007/ s00167-011-1510-4

4. Charous MT, Madison SJ, Suresh PJ, et al. Continuous femoral nerve blocks: varying local anesthetic delivery method (bolus versus basal) to minimize quadriceps motor block while maintaining sensory block. Anesthesiology. 2011;115(4):774-781. doi:10.1097/ALN.0b01 3e3182124dc6

5. Ilfeld BM, Duke KB, Donohue MC. The association between lower extremity continuous peripheral nerve blocks and patient falls after knee and hip arthroplasty. Anesth Analg. 2010;111(6):1552-1554. doi:10.1213/ANE.0b013e3181fb9507

6. Elkassabany NM, Antosh S, Ahmed M, et al. The risk of falls after total knee arthroplasty with the use of a femoral nerve block versus an adductor canal block: a double-blinded randomized controlled study. Anesth Analg. 2016;122(5):1696-1703. doi:10.1213/ANE.000 0000000001237

7. Brodner G, Buerkle H, Van Aken H, et al. Postoperative analgesia after knee surgery: a comparison of three different concentrations of ropivacaine for continuous femoral nerve blockade. Anesth Analg. 2007;105(1):256-262. doi:10.1213/01.ane.0000265552.43299.2b

8. Marhofer D, Kettner SC, Marhofer P, Pils S, Weber M, Zeitlinger M. Dexmedetomidine as an adjuvant to ropivacaine prolongs peripheral nerve block: a volunteer study. Br J Anaesth. 2013;110(3):438-442. doi:10.1093/bja/aes400

9. Jæger P, Grevstad U, Koscielniak-Nielsen ZJ, Sauter AR, Sørensen JK, Dahl JB. Does dexamethasone have a perineural mechanism of action? A paired, blinded, randomized, controlled study in healthy volunteers. Br J Anaesth. 2016;117(5):635-641. doi:10.1093/bja/aew318

10. El-Hennawy AM, Abd-Elwahab AM, Abd-Elmaksoud AM, ElOzairy HS, Boulis SR. Addition of clonidine or dexmedetomidine to bupivacaine prolongs caudal analgesia in children. $\mathrm{Br} J$ Anaesth. 2009;103(2):268-274. doi:10.1093/bja/aep159

11. Choi S, Rodseth R, McCartney CJ. Effects of dexamethasone as a local anaesthetic adjuvant for brachial plexus block: a systematic review and meta-analysis of randomized trials. $\mathrm{Br} J$ Anaesth. 2014;112(3):427-439. doi:10.1093/bja/aet417

12. Brummett CM, Hong EK, Janda AM, Amodeo FS, Lydic R. Perineural dexmedetomidine added to ropivacaine for sciatic nerve block in rats prolongs the duration of analgesia by blocking the hyperpolarization-activated cation current. Anesthesiology. 2011;115 (4):836-843. doi:10.1097/ALN.0b013e318221fcc9

13. Paauwe JJ, Thomassen BJ, Weterings J, van Rossum E, Ausems ME. Femoral nerve block using ropivacaine $0.025 \%, 0.05 \%$ and $0.1 \%$ : effects on the rehabilitation programme following total knee arthroplasty: a pilot study. Anaesthesia. 2008;63(9):948-953. doi:10.1111/ j.1365-2044.2008.05538.x

14. Ilfeld BM, Moeller LK, Mariano ER, et al. Continuous peripheral nerve blocks: is local anesthetic dose the only factor, or do concentration and volume influence infusion effects as well? Anesthesiology. 2010;112(2):347-354. doi:10.1097/ALN.0b013e3181ca4e5d 
15. Sharma B, Rupal S, Swami AC, Lata S. Effect of addition of dexmedetomidine to ropivacaine $0.2 \%$ for femoral nerve block in patients undergoing unilateral total knee replacement: a randomised double-blind study. Indian J Anaesth. 2016;60(6):403-408. doi:10. 4103/0019-5049.183392

16. Abdallah FW, Brull R. Facilitatory effects of perineural dexmedetomidine on neuraxial and peripheral nerve block: a systematic review and meta-analysis. Br J Anaesth. 2013;110(6):915-925. doi:10.1093/ bja/aet066

17. El-Boghdadly K, Brull R, Sehmbi H, Abdallah FW. Perineural dexmedetomidine is more effective than clonidine when added to local anesthetic for supraclavicular brachial plexus block: a systematic review and meta-analysis. Anesth Analg. 2017;124(6):2008-2020. doi:10.1213/ANE.0000000000002014

18. Brummett CM, Norat MA, Palmisano JM, Lydic R. Perineural administration of dexmedetomidine in combination with bupivacaine enhances sensory and motor blockade in sciatic nerve block without inducing neurotoxicity in rat. Anesthesiology. 2008;109(3):502-511. doi:10.1097/ALN.0b013e318182c26b

19. Packiasabapathy SK, Kashyap L, Arora MK, et al. Effect of dexmedetomidine as an adjuvant to bupivacaine in femoral nerve block for perioperative analgesia in patients undergoing total knee replacement arthroplasty: a dose-response study. Saudi J Anaesth. 2017;11 (3):293-298. doi:10.4103/sja.SJA_624_16
20. Mohamed SA, Fares KM, Mohamed AA, Alieldin NH. Dexmedetomidine as an adjunctive analgesic with bupivacaine in paravertebral analgesia for breast cancer surgery. Pain Physician. 2014;17(5):E589-598.

21. Seo SS, Kim OG, Seo JH, Kim DH, Kim YG, Park BY. Comparison of the effect of continuous femoral nerve block and adductor canal block after primary total knee arthroplasty. Clin Orthop Surg. 2017;9 (3):303-309. doi:10.4055/cios.2017.9.3.303

22. Herman T, Giladi N, Hausdorff JM. Properties of the 'timed up and go' test: more than meets the eye. Gerontology. 2011;57(3):203-210. doi:10.1159/000314963

23. Wiesmann T, Piechowiak K, Duderstadt S, et al. Continuous adductor canal block versus continuous femoral nerve block after total knee arthroplasty for mobilisation capability and pain treatment: a randomised and blinded clinical trial. Arch Orthop Trauma Surg. 2016;136(3):397-406. doi:10.1007/s00402015-2403-7

24. Jaeger P, Nielsen ZJ, Henningsen MH, Hilsted KL, Mathiesen O, Dahl JB. Adductor canal block versus femoral nerve block and quadriceps strength: a randomized, double-blind, placebo-controlled, crossover study in healthy volunteers. Anesthesiology. 2013;118(2):409-415. doi:10.1097/ALN.0b013e318279fa0b

\section{Publish your work in this journal}

The Journal of Pain Research is an international, peer reviewed, open access, online journal that welcomes laboratory and clinical findings in the fields of pain research and the prevention and management of pain. Original research, reviews, symposium reports, hypothesis formation and commentaries are all considered for publication. The manuscript management system is completely online and includes a very quick and fair peer-review system, which is all easy to use. Visit http:// www.dovepress.com/testimonials.php to read real quotes from published authors. 